Revista de Economia Política, vol. 21, nº 2 (82), pp. 304-321, abril-junho/2001

\title{
Taxa de juros, preferência pela liquidez e fundos de empréstimo: uma análise crítica das tentativas de demonstração da equivalência entre a teorias dos fundos de empréstimos e de preferência pela liquidez
}

\author{
Interest rate, liquidity preference and loan funds: a critical \\ analysis of attempts to demonstrate equivalence between \\ the theory of loan funds and preference for liquidity
}

JOSÉ LUÍS OREIRO*,**,

\begin{abstract}
RESUMO: Este artigo apresenta as tentativas de demonstrar a equivalência entre fundos de empréstimo e teorias de taxa de juros sobre preferências pela liquidez feitas por Hicks, Lerner, Tsiang e Patinkin, a fim de mostrar que essas tentativas não foram bem-sucedidas. Isso ocorre porque essas tentativas começaram com concepções erradas sobre quais são as questões em discussão no debate entre fundos de empréstimo e teorias de preferências pela liquidez ou porque elas deturparam uma ou outra das duas teorias. De fato, as tentativas de Hicks e Patinkin desconsideram o fato de que o essencial neste debate foi o mecanismo pelo qual as decisões de poupança e investimento influenciam na determinação da taxa de juros. Por outro lado, as tentativas de Lerner e Tsiang deturpam a relação entre poupança e investimento e o motivo financeiro da demanda por dinheiro que é suposto pelas duas teorias. PALAVRAS-CHAVE: Keynesianismo; taxa de juros; história do pensamento econômico; preferência pela liquidez.
\end{abstract}

ABSTRACT: This article presents the attempts to demonstrate the equivalence between loanable funds and liquidity preference theories of interest rate done by Hicks, Lerner, Tsiang and Patinkin in order to show that these attempts were not succeed. That is so because these attempts had started from wrong conceptions about what are the issues under discussion in the debate between loanable funds and liquidity preference theories or because they had misrepresented one or another of both theories. In fact, the attempts done by Hicks and Patinkin disconsider the fact that what was essential in this debate was the mechanism by which saving and investment decisions have influence in the determination of interest rate. On the

\footnotetext{
* Doutorando em Economia (IE-UFRJ) e Professor Assistente da Faculdade de Economia e Finanças do Instituto Brasileiro de Mercado de Capitais (Ibmec).

** O autor agradece aos comentários do Prof. Dr. Fernando Cardim de Carvalho a uma versão anterior do presente artigo. Eventuais falhas, no entanto, são de minha inteira responsabilidade.
} 
other hand, the attempts of Lerner and Tsiang misrepresent the relation between saving and investment and the finance motive of demand for money that is supposed by both theories. KEYWORDS: Keynesianism; interest rate; history of economic thought; liquidity preference. JEL Classification: B22; E12.

\section{INTRODUÇÃO}

Um importante debate que se seguiu à publicação da Teoria Geral - doravante TG - de Keynes refere-se aos determinantes da taxa de juros. A teoria prevalecente até aquele momento tinha sido a teoria dos fundos de empréstimo (T.F.E.), segundo a qual a taxa de juros seria determinada pela oferta e demanda de fundos emprestáveis. Essas forças de oferta e de demanda refletiriam variáveis de natureza tanto monetária - propensão ao entesouramento e política de crédito dos bancos - como variáveis de natureza real - produtividade do capital e abstinência das famílias. Na TG, contudo, Keynes apresentou uma nova teoria a respeito da determinação da taxa de juros, a teoria da preferência pela liquidez (T.P.L.). De acordo com essa teoria, a taxa de juros seria determinada pela oferta e demanda de moeda; ou, mais precisamente, na margem de indiferença entre a retenção de ativos líquidos (moeda) e ativos ilíquidos (obrigações). A taxa de juros seria, nesse caso, um fenômeno estritamente monetário.

Nesse contexto, a diferença entre a T.F.E. e a T.P.L. se expressaria na relação existente entre a eficiência marginal do capital/propensão a poupar e o nível da taxa de juros ${ }^{1}$. Para Keynes, a relação entre as variáveis em consideração seria apenas indireta. Nesse contexto, um aumento da eficiência marginal do capital e/ ou uma redução da propensão a poupar, por exemplo, teriam impacto inicial sobre o nível de renda e de emprego; mais precisamente, haveria um aumento do nível de emprego. Isso, por sua vez, produziria um aumento da demanda transacional por moeda, a qual - dada a política monetária e a preferência pela liquidez dos bancos - resultaria num aumento do nível da taxa de juros. Para os "Clássicos”, no entanto, as forças reais da produtividade do capital e da propensão a poupar teriam uma relação direta com a taxa de juros, isto é, o efeito inicial dos "choques" sobre as variáveis em questão seria um aumento do nível da taxa de juros. Esse aumento, por sua vez, induziria os indivíduos a reduzir o volume de encaixes monetários inativos - ou seja, haveria uma redução no entesouramento. Isso levaria a um excesso do investimento sobre a poupança, o qual - num contexto de preços e/ou salários nominais rígidos - induziria a um aumento do nível de renda e de emprego.

Uma análise atenta do debate entre Keynes e Robertson nos permite facilmente inferir que o ponto central no referido debate se constitui no mecanismo de

\footnotetext{
${ }^{1}$ Esse ponto é extensamente desenvolvido em Oreiro (1996, 1999a, 1999b).
} 
transmissão das flutuações da eficiência marginal do capital e da propensão a poupar sobre a taxa de juros. De fato, em carta endereçada a Robertson em 13/02/1936, Keynes diz que:

"I have many pages on the theme that increasing investment involves increasing output and that this hicks back on the rate of interest by draining away more money into active circulation, so that (... ) a high level of activity carries the seeds of its own destruction by raising interest too high" (CWJMK, vol. XIV, p. 91).

E Robertson contesta essa afirmação de Keynes dizendo que:

"Here I will only say that it seems to me a most misleading way of expressing the casual train of events to say, as it is sometimes done, that the act of trift lowers the rate of interest through lowering total incomes. I should say that it lowers the rate of interest quite directly through swelling the money stream of securities; that this fall in the rate of interest increases the proportion of resources over which people wish to keep command in monetary form and that this increase in tum is a cause of these being a net decline in total money incomes" (Robertson, 1940, p. 30).

Ao longo do tempo, porém, uma série de economistas passou a contestar a ideia de que existiriam diferenças relevantes entre a T.P.L. e a T.F.E. Em outros termos, passou-se a argumentar que ambas seriam formas alternativas de apresentar os fatores determinantes da taxa de juros; sem, contudo, alterar natureza de tais fatores, ou seja, as referidas teorias seriam formas equivalentes de determinação da taxa de juros. Essa linha de interpretação do debate entre a T.F.E. e a T.P.L. foi defendida por Hicks (1939), Lerner (19476), Tsiang (1956/80) e Patinkin (1958). Nas palavras de Hicks:

"A taxa de juros é determinada pela oferta e procura de fundos de financiamento, ou é determinada pela oferta e procura do próprio dinheiro? (...) Espero provar que não faz a menor diferença essa forma de apresentar a questão ou se seguirmos os autores que adotam no presente momento o ponto de vista contrário. Seguidas adequadamente, as duas abordagens levam aos mesmos resultados” (Hicks, 1987, p. 129).

O presente artigo tem como objetivo analisar as tentativas de demonstração da equivalência entre a T.F.E. e a T.P.L. que se seguiram à contribuição original de Hicks para o tema em questão. Nesse contexto, procuraremos demonstrar que:

As sucessivas tentativas de demonstração da equivalência entre a T.F.E. e a T.P.L. partiam de concepções completamente diferentes a respeito de quais seriam os pontos de divergência entre as referidas teorias. De fato, podemos identificar ao menos três diferentes concepções a respeito da natureza de tais divergências, a saber: o mercado no qual a taxa de juros é determinada, a dicotomia estoques versus 
fluxos e as relações existentes entre poupança, investimento e demanda de moeda. Todas as tentativas de demonstração da equivalência entre as referidas teorias, sendo Tsiang $(1956,1981)$ a única exceção, desconsideraram a questão que era o cerne do debate entre Keynes e os clássicos a respeito da determinação da taxa de juros, qual seja, o mecanismo pelo qual a eficiência marginal do capital e a propensão a poupar tem influência sobre o nível de taxa de juros. Segue-se, portanto, que tais tentativas de demonstração da equivalência entre a T.F.E. e a T.P.L. são inválidas, uma vez que não tratam da questão que é o ponto fundamental de discórdia entre os defensores de ambas as teorias. No que se refere a demonstração de equivalência feita por Tsiang, a mesma também é inválida porque desconsidera o tratamento dado por Keynes ao motivo finanças de demanda de moeda, qual seja, o de fundo rotativo de ativos líquidos.

Dadas essas considerações, o presente artigo está estruturado da seguinte forma. A segunda seção apresenta a demonstração de Hicks da equivalência entre a T.F.E. e a T.P.L. A terceira seção apresenta as tentativas pós-Hicks de demonstração da equivalência entre as referidas teorias. A quarta seção está dedicada à avaliação do debate acerca da equivalência entre a T.F.E. e a T.P.L. A quinta seção sumariza as conclusões obtidas ao longo do presente artigo.

\section{A "SÍNTESE” DE HICKS: A IRRELEVÂNCIA DO DEBATE ENTRE T.F.E. E T.P.L.}

Segundo Hicks o debate entre a T.P.L. e a T.F.E. partia de uma falsa concepção a respeito de como os preços e, em particular, a taxa de juros são determinados numa economia de mercado. A interdependência entre os diversos setores da economia garante que o preço de qualquer bem ou serviço seja determinado em conjunto com os preços das demais bens e serviços existentes no sistema econômico (cf. Hicks, 1987, p. 130).

Nesse contexto, a T.F.E. e a T.P.L. estariam propondo que existe um mercado particular no qual a taxa de juros é determinada. A T.F.E. estaria propondo a determinação da taxa de juros pela interação entre demanda e oferta de obrigações; ao passo que a T.P.L. estaria propondo a referida determinação por intermédio da interação entre demanda e oferta de moeda. Visto sob a perspectiva do Equilíbrio Geral, o debate entre a T.P.L. e a T.F.E. perde totalmente o significado: pode-se provar que tanto o mercado de obrigações como o mercado desempenham igual papel na determinação da taxa de juros; bem como demonstrar que a T.F.E. e a T.P.L., se corretamente especificadas - ou seja, se apresentadas num contexto de equilíbrio geral, em lugar de equilíbrio parcial - são apenas formas equivalentes de apresentar a determinação da taxa de juros (cf. Hicks, 1987, p. 129).

Para demonstrar esse ponto, Hicks considera uma economia ancorada no método sequencial Robertsoniano, onde existem n bens e serviços, moeda e obri- 
gações ${ }^{2}$. Ao todo são $\mathrm{n}+2$ mercados. No início de cada período os indivíduos possuem uma determinada dotação de moeda e obrigações que herdaram da semana anterior. Chamemos de $\mathrm{M}_{\mathrm{t}, \mathrm{i}}$ e $\mathrm{B}_{\mathrm{t}, \mathrm{i}}$ as quantidades iniciais de títulos e moeda possuídas pelo "i-ésimo" indivíduo.

A economia em consideração é uma economia de troca pura, de forma que as quantidades disponíveis de cada uma das $n$ mercadorias é tida como constante. No início de cada período, as famílias recebem uma determinada quantidade dessas mercadorias como dotação para o período. Tomando os preços dessas $n$ mercado rias como dados - concorrência perfeita - as famílias têm que alocar o valor de suas dotações de mercadorias, moeda e obrigações de forma que a seguinte restrição orçamentária seja satisfeita:

$$
\mathrm{M}_{\mathrm{t}+1, \mathrm{i}}+\mathrm{B}_{\mathrm{t}+1, \mathrm{i}}+\mathrm{p}_{\mathrm{j}} \cdot \mathrm{X}_{\mathrm{j}, \mathrm{i}}=\mathrm{M}_{\mathrm{t}, \mathrm{i}}+\mathrm{B}_{\mathrm{t}, \mathrm{i}}+\sum \mathrm{p}_{\mathrm{j}} \cdot \mathrm{W}_{\mathrm{j}, \mathrm{i}} \quad \forall \mathrm{i}=1, \ldots, \mathrm{m}
$$

onde: $\mathrm{M}_{\mathrm{t}+1, \mathrm{i}}$ é a quantidade de moeda que o indivíduo i deseja ter ao final do período $\mathrm{t} ; \mathrm{B}_{\mathrm{t}+1, \mathrm{i}}$ é a quantidade de títulos que o indivíduo i deseja ter ao final do período t; $\mathrm{X}_{\mathrm{j}, \mathrm{i}}$ é a quantidade da mercadoria $\mathrm{j}$ que o indivíduo i deseja consumir em $\mathrm{t}$; $\mathrm{W}_{\mathrm{j}, \mathrm{i}}$ é a quantidade da mercadoria $\mathrm{j}$ que o indivíduo i possui em $\mathrm{t}$.

Se agregarmos as restrições orçamentárias dos $m$ indivíduos dessa economia, temos que:

$$
\mathrm{M}_{\mathrm{t}+1}+\mathrm{B}_{\mathrm{t}+1}+\sum \mathrm{p}_{\mathrm{j}} \cdot \mathrm{X}_{\mathrm{j}, \mathrm{i}}=\mathrm{M}_{\mathrm{t}}+\mathrm{B}_{\mathrm{t}}+\sum \mathrm{p}_{\mathrm{j}} \cdot \mathrm{W}_{\mathrm{j}}
$$

O equilíbrio em cada um dos $n+2$ mercados é dado pelas condições que se seguem:

$$
\begin{aligned}
& \mathrm{X}_{\mathrm{j}}\left(\mathrm{p}_{1} / \mathrm{p}, \ldots, \mathrm{p}_{\mathrm{i}} / \mathrm{p}, \ldots, \mathrm{p}_{\mathrm{n}} / \mathrm{p}, \mathrm{r}, \sum,\left(\mathrm{p}_{\mathrm{j}} / \mathrm{p}\right) \mathrm{W}_{\mathrm{j}}+\mathrm{M}_{\mathrm{t}} / \mathrm{p}+\mathrm{B}_{\mathrm{t}} / \mathrm{p}\right)=\mathrm{W}_{\mathrm{j}} ; \mathrm{j}=1, \ldots, \mathrm{n} \\
& \mathrm{M}_{\mathrm{t}+1}\left(\mathrm{p}_{1} / \mathrm{p}, \ldots, \mathrm{p}_{\mathrm{i}} / \mathrm{p}, \ldots, \mathrm{p}_{\mathrm{n}} / \mathrm{p}, \mathrm{r}, \sum\left(\mathrm{p}_{\mathrm{j}} / \mathrm{p}\right) \mathrm{W}_{\mathrm{j}}+\mathrm{M}_{\mathrm{t}} / \mathrm{p}+\mathrm{B}_{\mathrm{t}} / \mathrm{p}\right)=\mathrm{M}_{\mathrm{t}} \\
& \mathrm{B}_{\mathrm{t}+1}\left(\mathrm{p}_{1} / \mathrm{p}, \ldots, \mathrm{p}_{\mathrm{i}} / \mathrm{p}, \ldots, \mathrm{p}_{\mathrm{n}} / \mathrm{p}, \mathrm{r}, \sum\left(\mathrm{p}_{\mathrm{j}} / \mathrm{p}\right) \mathrm{W}_{\mathrm{j}}+\mathrm{M}_{\mathrm{t}} / \mathrm{p}+\mathrm{B}_{\mathrm{t}} / \mathrm{p}\right)=\mathrm{B}_{\mathrm{t}} \\
& \mathrm{P}={ }_{1} \mathrm{p}_{1}+\Theta_{2} \mathrm{p}_{2}+\ldots+\Theta_{\mathrm{n}} \mathrm{p}_{\mathrm{n}}
\end{aligned}
$$

onde: $\Theta_{\mathrm{t}}$ é a participação do bem i no nível geral de preços.

De (3)-(6) temos $n+3$ equações para determinar $n+2$ incógnitas: os preços relativos $(\mathrm{p} / \mathrm{p})$, a taxa de juros real (r), e o nível geral de preços (p). Entretanto, pela equação (2) apenas $n+2$ dessas equações são linearmente independentes: a equação restante pode ser escrita como uma combinação linear das demais.

Nesse contexto, se eliminarmos a equação de equilíbrio do mercado de obrigações, a taxa de juros será determinada pela condição de equilíbrio do mercado monetário em conjunto com as equações de equilíbrio dos outros n mercados. Essa configuração para o sistema apresentado seria, para Hicks, a correta especificação da teoria da preferência pela liquidez. Paralelamente, se escolhermos por eliminar a equação de equilíbrio do mercado monetário; a taxa de juros será determinada pela condição de equilíbrio do mercado de obrigações em conjunto com

\footnotetext{
${ }^{2}$ O modelo apresentado a seguir tem por base Harris (1981).
} 
as demais $n$ equações do sistema. Teremos, então, a T.F.E. corretamente especifica$\mathrm{da}^{3}$. Como em ambos os casos a taxa de juros é a mesma, segue-se que as teorias em questão seriam apenas formas equivalentes de se apresentar a determinação da taxa de juros. Nas palavras de Hicks:

"Se quisermos, podemos eliminar a equação do dinheiro, determinando assim os preços das mercadorias e a taxa de juros através da demanda e oferta de fundos de empréstimos (...) Como alternativa, podemos seguir Keynes e eliminar a outra equação(...) Se isso for feito, os n-1 preços habituais e a única taxa de juros serão determinados pelas equações de demanda e oferta de $\mathrm{n}$ mercadorias, incluindo o dinheiro...

A mim parece que ambos os métodos são perfeitamente legítimos; a escolha entre eles é apenas uma questão de conveniência" (ibid., p. 134).

Paralelamente, a interdependência entre as equações que compõem o modelo apresentado garantiria que a taxa de juros é influenciada tanto pelas condições de oferta e demanda de moeda, como pela oferta e demanda de obrigações. Sendo assim, o debate entre a T.F.E. e a T.P.L. não teria sentido, já que as forças de oferta e de demanda enfatizadas por ambas as teorias desempenham igual papel na determinação da taxa de juros. Keynes e seus críticos só não chegaram a essa conclusão por ancorarem suas respectivas análises a respeito da determinação da taxa de juros no método de equilíbrio parcial marshalliano.

\section{TENTATIVAS PÓS-HICKS DE DEMONSTRAÇÃO DA EQUIVALÊNCIA ENTRE A T.F.E. E A T.P.L.}

A posição de Hicks foi aceita pela maior parte dos economistas, que passaram a acreditar que nenhum ponto de importância teórica fundamental estava envolvido no debate entre as referidas teorias (cf. Carvalho, 1994, p. 117). Apesar do consenso que se seguiu após a contribuição de Hicks, alguns autores tornaram a levantar o problema da equivalência entre a T.F.E. e a T.P.L. Tais autores passaram a questionar a validade da demonstração de equivalência entre as teorias em questão dada pelo referido autor; ou, pelo menos, a abrangência da mesma.

Entre tais autores, os mais destacados foram A. Lerner (19476), S. C. Tsiang (1956/80) e Patinkin (1958). Cada um dos mesmos reafirmou a conclusão de Hicks de que as teorias em consideração são formas equivalentes de apresentar a determinação da taxa de juros. A diferença relativamente a Hicks é que, para eles, o cerne do debate entre a T.F.E. e a T.P.L. não consistia em saber qual o mercado no qual a taxa de juros é determinada; mas estaria centrado em outros pontos, como, por exemplo, a relação entre poupança e investimento, a dicotomia estoques-fluxos e etc.

\footnotetext{
${ }^{3}$ Essa interpretação da análise de Hicks tem por base Patinkin (1958).
} 
A demonstração de A. Lerner: a igualdade entre poupança e investimento.

A primeira tentativa de reconciliação entre as duas teorias, pós-Hicks, foi feita por Abba Lerner. Em lugar de se concentrar nas implicações da Lei de Walras, como fez Hicks, Lerner enfatizou a ideia apresentada na TG de que poupança e investimento seriam magnitudes necessariamente iguais; de forma que não desempenhariam nenhum papel na determinação da taxa de juros, embora desempenhem um papel importante na determinação do nível de renda.

$\mathrm{O}$ investimento se constitui na parte do gasto que não foi destinado a compra de bens de consumo, ou seja: I = E - C (7); onde: E é o dispêndio agregado.

A poupança continua sendo definida como a parte da renda que não foi gasta em consumo. Para estabelecer a igualdade necessária entre poupança e investimento basta que se considere que o gasto será idêntico à renda. Nesse caso, temos: $\mathrm{I}=\mathrm{Y}-\mathrm{C}=\mathrm{S}(8)$.

De acordo com Lerner, a identidade entre dispêndio e renda seria verdadeira, uma vez que:

"The sum of the incomes of all the individuals in the economy, $\mathrm{Y}$, is equal to the sum of the expenditures of all kinds by the individuals of the economy, since these expenditures are nothing but the payments; the receipts of which constitutes all the incomes" (Cf. Lerner, 1947a, p. 620).

Deve-se observar, com base na citação acima, que Lerner considera que a renda é idêntica ao dispêndio porque está considerando a renda e o dispêndio ex-post. Todo o gasto, uma vez efetivado, gera uma renda em igual magnitude, isso porque todo o pagamento equivale a um recebimento, dado o caráter bilateral de qualquer transação mercantil (cf. Possas, 1987, p. 51). Nesse caso, a poupança e o investimento serão necessariamente iguais, como se observa pelas equações apresentadas anteriormente. Contudo, como a renda e o dispêndio aqui considerados se constituem na renda e no dispêndio ex-post, a poupança e o investimento, de que se trata aqui, também serão ex-post.

Uma vez demonstrada a igualdade necessária entre poupança e investimento, Lerner utiliza esta identidade para afirmar que as curvas de poupança e investimento, que seriam um dos conjuntos de determinantes da taxa de juros de acordo com a teoria de fundos de empréstimo, coincidem entre si (Figura 1) ao longo de todo plano $<$ r, S,I $>$.

Em outros termos, não existe uma curva de poupança independente da curva de investimento, mas as duas curvas são, de fato, uma única curva, a curva $S=1$. Essa curva $S=I$ demonstra que para cada nível particular de taxa de juros, o total poupado será igual ao total investido (ibid., p. 640). 


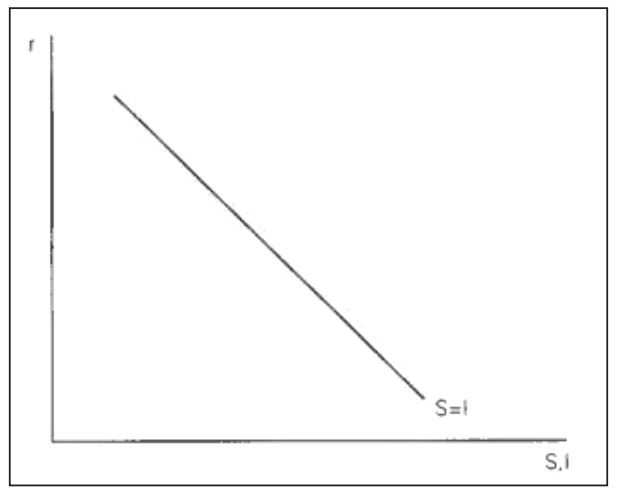

Para estabelecer a equivalência entre as teorias de fundos de empréstimo e de preferência pela liquidez, só resta demonstrar que os demais componentes da demanda e da oferta de fundos de empréstimo, a saber: a criação de crédito e a variação do entesouramento podem ser reescritos em termos de demanda e oferta de moeda como estoque. A condição de equilíbrio no mercado de fundos de empréstimo é dada por:

$$
\mathrm{I}_{\mathrm{t}}+\mathrm{DH}_{\mathrm{t}}=\mathrm{S}_{\mathrm{t}}+\mathrm{DM}_{\mathrm{t}}
$$

onde: $\mathrm{DH}_{t}$ é a variação do entesouramento em t; $\mathrm{DM}_{t}$ é a variação da oferta de moeda em t.

Como, da discussão procedente, temos que $\mathrm{II}_{t}=\mathrm{S}_{\mathrm{t}}$, segue-se que a condição de equilíbrio no mercado de fundos de empréstimo pode ser dada por:

$\mathrm{DH}_{\mathrm{t}}=\mathrm{DM}_{\mathrm{t}}$

Entretanto, sabemos que:

$$
\begin{aligned}
& \mathrm{DH}_{\mathrm{t}}=\mathrm{H}_{\mathrm{t}}-\mathrm{H}_{\mathrm{t}-1} \\
& \mathrm{DM}_{\mathrm{t}}=\mathrm{M}_{\mathrm{t}}-\mathrm{M}_{\mathrm{t}-1}
\end{aligned}
$$

onde: $H_{t}$ é a quantidade de moeda que os agentes desejam reter em t; $M_{t}$ é a quantidade de moeda disponível no tempo t.

Como no início do período $t$, temos que $\mathrm{H}_{\mathrm{t}-1}=\mathrm{M}_{\mathrm{t}-1, \mathrm{uma}}$ vez que o dinheiro existente na economia deve estar sendo retido por algum agente; segue-se que se (10) estiver sendo atendida, então $M_{t}$ deve ser igual a $H_{t}$ ou seja, a demanda e a oferta de moeda como estoque também serão iguais. Conclui-se, portanto, que a teoria dos fundos de empréstimo, se corretamente especificada, ou seja, se levar em conta a identidade necessária entre as decisões de poupança e investimento, é equivalente à teoria da preferência pela liquidez.

Lerner apresenta também uma demonstração geométrica da equivalência entre ambas as teorias. Tal demonstração pode ser acompanhada pela Figura 2. 


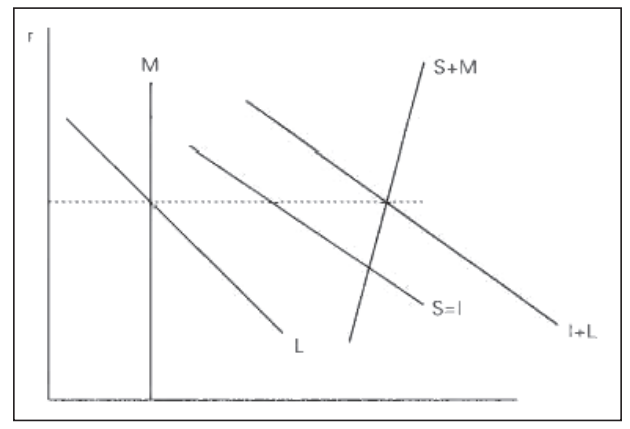

O canto esquerdo da figura 2 apresenta a T.P.L., ou seja, a determinação da taxa de juros por intermédio da demanda e da oferta de moeda (curvas L e M respectivamente). $\mathrm{Na}$ extremidade direita do gráfico visualiza-se a determinação da taxa de juros por meio da demanda e da oferta de fundos de empréstimos (curvas $\mathrm{I}+\mathrm{L}$ e $\mathrm{S}+\mathrm{M}$, respectivamente).

A lógica da demonstração geométrica é simples: a curva $S=I$ é somada ponto a ponto às curvas $\mathrm{M}$ e L. Como resultado se obtêm as curvas $\mathrm{I}+\mathrm{L}$ e $\mathrm{S}+\mathrm{M}$, correpondentes à demanda e à oferta de crédito. Como não pode haver divergência entre poupança e investimento, segue-se que o resultado dessa adição não pode mudar a taxa de juros que equilibra a demanda e a oferta de moeda. Daqui se conclui que a T.P.L. e a T.F.E. são formas equivalentes de apresentar a determinação da taxa de juros.

\section{A redescoberta do motivo finanças: Tsiang (1956/1980)}

Uma segunda tentativa de reconciliação entre as teorias em questão foi feita por Tsiang $(1956,1980)$. Esse autor tenta demonstrar a equivalência entre as duas teorias provando que as decisões de demanda e de oferta de fundos de empréstimo envolvem necessariamente decisões correspondentes de demanda e de oferta de moeda. Dito de outra forma, os argumentos das funções demanda e oferta de moeda seriam as próprias funções de demanda e de oferta de fundos de empréstimo.

Para demonstrar esse ponto, Tsiang considera uma economia na qual o tempo se encontra dividido em uma série de intervalos discretos, que são tão pequenos ao ponto de que a renda recebida num intervalo qualquer t só pode ser gasta no intervalo seguinte $\mathrm{t}+\mathrm{l}$. Ao início do período $\mathrm{t}$, os indivíduos dessa economia possuem em cash a totalidade da renda recebida no período t-1 mais os encaixes ociosos retidos em t-1. Essa disponibilidade monetária deverá ser alocada entre gastos de consumo, compra das obrigações que as firmas emitem para financiar seus projetos de investimento e encaixes ociosos para o período em consideração. Deve-se observar também que, como a renda que é ganha pelos indivíduos no período t não pode ser gasta em t, a restrição orçamentária dos indivíduos dessa economia corresponde a uma restrição do tipo "cash-in-advance"; que pode ser expressa pela seguinte equação:

$$
M_{t}=Y_{t-1}+H_{t-1}=P_{t} \cdot c_{t}+B_{t, h}+H_{t}
$$


onde: $\mathrm{M}_{\mathrm{t}}$ é a disponibilidade de moeda em $\mathrm{t} ; \mathrm{Y}_{\mathrm{t}-1_{1}}$ é a renda monetária obtida em t-1; $\mathrm{H}_{\mathrm{t}-1}$ é a quantidade de moeda retida voluntariamente em $\mathrm{t}-1 ; \mathrm{P}_{\mathrm{t}}$ é o nível geral de preços em t; $c_{t}$ é o consumo real em $t ; B_{t, h}$ é a demanda de obrigações pelas famílias em $t$.

A totalidade de moeda retida pelos indivíduos no início do período t corresponde à oferta de moeda no período t-l $\left(\mathrm{Ms}_{\mathrm{t}-\mathrm{-}}\right)$; ou seja, no início do período t os indivíduos possuem a totalidade do estoque de moeda existente na economia. Sendo assim, temos que:

$$
\mathrm{M}_{\mathrm{t}=} \mathrm{M}_{\mathrm{s}, \mathrm{t}-1}
$$

Observe-se, também, que (12) pode ser reescrita da seguinte forma:

$$
\mathrm{B}_{\mathrm{t}, \mathrm{h}}=\left(\mathrm{Y}_{\mathrm{t}-1}-\mathrm{C}_{\mathrm{t}}\right)+\left(\mathrm{H}_{\mathrm{t}-1}-\mathrm{H}_{\mathrm{t}}\right)
$$

onde: $C_{t}$ é o consumo nominal em $t\left(C_{t}=p_{t} \cdot c_{t}\right)$

Em palavras: a demanda de obrigações por parte das famílias, que corresponde a uma parte da oferta de fundos de empréstimo, é igual à poupança planejada mais o desentesouramento líquido para o período em consideração.

A oferta de fundos de empréstimo $\left(S_{t}\right)$ consiste na demanda de obrigações por parte das famílias mais a injeção de moeda feita pelos bancos comerciais e pelas autoridades monetárias no início do período t. Temos então que:

$$
\mathrm{S}_{\mathrm{t}=\mathrm{Bt}, \mathrm{h}}+\left(\mathrm{M}_{\mathrm{t}}-\mathrm{M}_{\mathrm{t}-1)}\right.
$$

A demanda por fundos de empréstimo consiste na totalidade dos projetos de investimento que as firmas desejam implementar no tempo t. Isso porque se supõe que as firmas não possuem lucros retidos para financiar ais projetos, e que os consumidores não possuem nenhum tipo de acesso ao crédito, ou seja, os mesmos não podem tomar empréstimos para financiar seus gastos em consumo. Temos, então, que:

$$
\mathrm{B}_{\mathrm{t}, \mathrm{f}}=\mathrm{D}_{\mathrm{t}}=\mathrm{I}_{\mathrm{t}}
$$

onde: $\mathrm{D}_{\mathrm{t}}$ é a demanda de fundos de empréstimo em $\mathrm{t}$; $\mathrm{B}_{\mathrm{t}, \mathrm{f}}$ é o volume de obrigações emitidas pelas firmas em t; $\mathrm{I}_{t}$ é o investimento nominal em $t$.

Em equilíbrio, a demanda e a oferta de fundos de empréstimo devem ser iguais, ou sela:

$$
I_{t}=Y_{t-1}-C_{t}-H_{t}+H_{t-1}+M_{t}-M_{t-1}
$$

Como de (12) temos que $\mathrm{Y}_{\mathrm{t}-1}+\mathrm{H}_{\mathrm{t}-1}=\mathrm{M}_{\mathrm{t}}$; de (13) $\mathrm{M}_{\mathrm{t}}=\mathrm{M}_{\mathrm{s}, \mathrm{t}-1}$; e sabendo que $\mathrm{M}_{\mathrm{t}-1}$ corresponde à oferta de moeda no tempo $t$, temos que $\mathrm{M}_{\mathrm{t}-1}=\mathrm{H}_{\mathrm{t}-1}+\mathrm{Y}_{\mathrm{t}-1}$. Utilizando essa relação em (17) temos que:

$$
\mathrm{M}_{\mathrm{t}}=\mathrm{H}_{\mathrm{t}}+\mathrm{I}_{\mathrm{t}}+\mathrm{C}_{\mathrm{t}}
$$

A equação (18) corresponde ao equilíbrio no mercado monetário, ou seja, a demanda de moeda, que consiste nos encaixes ativos - utilizados para financiar os gastos de consumo e os gastos de investimento - e nos encaixes inativos; deve, em equilíbrio, ser igual à oferta de moeda para o período. Segue-se, portanto, que o equilíbrio no mercado de fundos de empréstimo implica um equilíbrio no mercado 
monetário; e, mais importante, a demanda por moeda é governada pelos mesmos determinantes das funções de demanda e oferta de fundos de empréstimos. Nas palavras de Tsiang:

"Thus, the liquidity preference and loanable funds theories are indeed two different ways of saying the same thing, leaving in all conceivable circumstances to the same result. The perfect agreement between the two theories is found to exist because the ex-ante decisions to supply loanable funds to the market necessarily imply corresponding decisions as to the funds required to finance one's own consumption and one's own demand for idle money; while the decisions to take loanable funds off the market necessarily imply corresponding decisions as to the requirements for funds to finance investment expenditures" (1956, p. 60).

Como se vê na citação acima, Tsiang atribui a equivalência entre as teorias de fundos de empréstimo e de preferência pela liquidez, a demonstração de que toda a decisão de oferta e de demanda de fundos de empréstimo corresponde a uma decisão de demanda e de oferta de moeda. Essa correspondência seria originada pelo fato de que as demandas de consumo e de investimento, devido à hipótese de "cash-in-advance", têm que ser financiadas com a aquisição prévia de encaixes monetários. Sendo assim, toda a decisão de demandar fundos de empréstimo - para a realização de investimento - corresponderia a uma decisão de demandar moeda; ao passo que toda a decisão de ofertar fundos de empréstimo - seja através da abstenção de consumo ou de redução dos encaixes ociosos - corresponderia a uma redução da demanda por moeda.

Para Tsiang (1980), Keynes afirmou na TG que as teorias de preferência pela liquidez e de fundos de empréstimo seriam teorias diferentes para a determinação da taxa de juros; porque desconsiderou a necessidade de financiamento prévio dos gastos de investimento (e dos gastos de consumo) por intermédio de aquisição prévia de encaixes monetários. Em outros termos, na TG Keynes desconsiderou o que mais tarde ele, no seu debate com Ohlin e Robertson, chamaria de motivo finanças de demanda por moeda (cf. Tsiang, 1980, p. 468).

A consideração do motivo finanças de demanda por moeda, segundo Tsiang, nos obriga a colocar o consumo planejado e o investimento planejado como argumentos da função de demanda por moeda. Nesse caso, essa função pode ser escrita da seguinte forma:

$$
\mathrm{M}_{\mathrm{d}, \mathrm{t}}=\mathrm{L}\left(\mathrm{C}_{\mathrm{p}, \mathrm{t}}+\mathrm{I}_{\mathrm{p}, \mathrm{t}}, \mathrm{r}_{\mathrm{t}}\right) ; \mathrm{L}_{1}>0, \mathrm{~L}_{2}<0
$$

onde: $\mathrm{M}_{\mathrm{d}, \mathrm{t}}$ é a demanda por moeda no tempo t; $\mathrm{C}_{\mathrm{p}, \mathrm{t}}$ é a demanda planejada de consumo no tempo t; $\mathrm{I}_{\mathrm{p}, \mathrm{t}}$ é a demanda planejada de investimento no tempo t; r é a taxa de juros nominal em $t$.

A condição de equilíbrio no mercado monetário pode então ser apresentada por:

$$
\mathrm{M}_{\mathrm{t}}=\mathrm{L}\left(\mathrm{C}_{\mathrm{p}, \mathrm{t}}+\mathrm{I}_{\mathrm{p}, \mathrm{t}} ; \mathrm{r}\right)
$$


Se linearizarmos (20) em torno do valor observado da taxa nominal de juros no período anterior, utilizando apenas os primeiros termos da expansão de Taylor; e supondo que a demanda por moeda é linear nos gastos planejados, temos que:

$$
\mathrm{L}\left(\mathrm{C}_{\mathrm{p}, \mathrm{t}}+\mathrm{I}_{\mathrm{p}, \mathrm{t}} ; \mathrm{r}\right)=\mathrm{K}\left(\mathrm{r}_{\mathrm{t}-1}\right) \cdot\left[\mathrm{C}_{\mathrm{p}, \mathrm{t}}+\mathrm{I}_{\mathrm{p}, \mathrm{t}}\right]
$$

Para o período t-1, a condição de equilíbrio no mercado monetário pode ser apresentada por:

$$
\mathrm{M}_{\mathrm{t}-1}=\mathrm{K}\left(\mathrm{r}_{\mathrm{t}-1}\right)\left[\mathrm{C}_{\mathrm{t}-1}+\mathrm{I}_{\mathrm{t}-1}\right]
$$

Substituindo (21) em (20), e operando a diferença entre a equação resultante e (22), temos que:

$$
\mathrm{DM}_{\mathrm{t}-1}=\mathrm{K}_{\mathrm{t}-1}\left(\mathrm{r}_{\mathrm{t}-1}\right) \cdot \mathrm{I}_{\mathrm{p}, \mathrm{t}}+\mathrm{K}_{\mathrm{t}-1}\left(\mathrm{r}_{\mathrm{t}-1}\right) \mathrm{S}_{\mathrm{p}, \mathrm{t}}+\mathrm{L}_{\mathrm{r}} \mathrm{Dr}_{\mathrm{t}}
$$

onde: $S_{p, t}$ é a poupança planejada em $t$, que é igual a $\left(\mathrm{Y}_{\mathrm{t}-1}-\mathrm{C}_{\mathrm{p}, \mathrm{t}}\right)$

De acordo com Tsiang, a equação (23) consiste na condição de equilíbrio do mercado de fundos de empréstimos, tal como apresentada por Robertson (Cf. Tsiang, 1980, p. 473). Por (23), observamos que os níveis de investimento e de poupança planejados pelas firmas e pelas famílias têm influência direta sobre a taxa de juros, independentemente de qualquer variação do nível de renda. Segue-se, portanto, que a taxa de juros, nesse caso, não é um fenômeno estritamente monetário, tal como defendido por Keynes na TG (cf. Tsiang, 1980, p. 474).

\section{A demonstração de Patinkin: o equilíbrio de estoques e o equilíbrio de fluxos}

Uma outra tentativa de demonstração de equivalência entre a T.F.E. e a T.P.L. foi realizada por Don Patinkin em um artigo publicado na Economica em 1958.

De acordo com Patinkin, a demonstração de equivalência entre as referidas teorias, dada por Hicks, era essencialmente correta; ou seja, ambas, se apresentadas num contexto de equilíbrio geral, seriam apenas formas alternativas de determinação da taxa de juros.

Entretanto, a argumentação de Hicks não havia sido suficientemente abrangente para demonstrar que não existia nenhuma diferença de caráter fundamental entre as teorias em consideração. De fato, diz Patinkin, restava ainda a objeção de que a T.F.E. impõe que a taxa de juros é determinada por variáveis de fluxo, ao passo que a T.P.L. considera que a mesma é determinada por variáveis de estoque. Em particular, a apresentação da T.F.E. dada por Hicks não seria correta: a condição de equilíbrio relevante para a dita teoria não seria a referente a igualdade entre os estoques demandados e ofertados de obrigações, mas a referente a igualdade entre os fluxos de demanda e oferta das mesmas.

Sendo assim, a aplicação da Lei de Walras, que permite a eliminação do mercado de obrigações ou a eliminação do mercado monetário, não demonstraria a equivalência entre as teorias em consideração. Isso porque, a essência do debate em questão estaria, não na escolha do mercado no qual a taxa de juros é determinada, mas em saber se a mesma é determinada por fluxos ou por estoques. A eli- 
minação do mercado de obrigações, nesse caso, apenas demonstraria que a T.P.L. pode ser igualmente formulada em termos do mercado de obrigações ou em termos do mercado monetário.

Com base nessas considerações, Patinkin tenta estender a análise de Hicks de forma a demonstrar que se os estoques desejados de demanda e oferta de obrigações estiverem em equilíbrio; então os fluxos demandados e ofertados dos mesmos também estarão. Em outras palavras, pode-se escrever, no sistema de equilíbrio geral apresentado por Hicks, a equação de equilíbrio do mercado de obrigações tanto em termos de estoques como em termos de fluxos (cf. Patinkin, 1958, p. 303).

Nesse caso, as conclusões obtidas por Hicks não dependeriam da forma pela qual se apresente a condição de equilíbrio no mercado de obrigações. Isso porque a equivalência entre o equilíbrio de estoques e o equilíbrio de fluxos garante que a Lei de Walras pode ser igualmente aplicada para ambos os casos; ou seja, pode-se descartar a condição de equilíbrio do mercado de obrigações no caso em que a mesma é escrita em termos de estoques ou no caso em que a mesma é escrita em termos de fluxos, sem que isso altere os preços e a taxa de juros de equilíbrio. Em outros termos, a dicotomia estoques-fluxos é irrelevante para o problema da determinação da taxa de juros.

Para demonstrar esse ponto, Patinkin considera uma economia análoga à descrita por Hicks: trata-se de uma economia de "troca-pura", na qual o tempo é dividido em intervalos discretos, denominados "semanas". No início de cada semana, os indivíduos procurarão definir os planos de compra e venda de mercadorias, os empréstimos a tomar e a conceder e a retenção de moeda ao final da semana.

No início da semana $t$, cada indivíduo possui um estoque de moeda igual a $\mathrm{M}_{\mathrm{t}-1}$, um estoque de obrigações igual a $\mathrm{B}_{\mathrm{t}-1,{ }_{-1}}$ e uma dotação de mercadorias igual $\mathrm{a}_{\mathrm{t}}$. Os planos de retenção de moeda e obrigações para o final do período $t$, e os planos de consumo para o período em consideração devem ser tais que obedeçam à seguinte restrição orçamentária;

$$
\mathrm{M}_{\mathrm{t}-1, \mathrm{i}}+\mathrm{B}_{\mathrm{t}-1, \mathrm{i}}+\mathrm{p}_{\mathrm{t}} \mathrm{x}_{\mathrm{t}, \mathrm{i}}=\mathrm{p}_{\mathrm{t}} \cdot \mathrm{c}_{\mathrm{t}, \mathrm{i}}+\mathrm{M}_{\mathrm{t}, \mathrm{i}}+\mathrm{B}_{\mathrm{t}, \mathrm{i}} \mathrm{i}=1, \ldots, \mathrm{n}
$$

De (24) podemos obter o estoque demandado de obrigações $-\mathrm{B}_{\mathrm{t}, 1} \cdot$ - como uma função da oferta líquida de bens e da oferta líquida de moeda. Temos, então, que:

$$
\mathrm{B}_{\mathrm{t}, \mathrm{i}}=\mathrm{g}_{\mathrm{s}, \mathrm{t}}-\mathrm{Dm}_{\mathrm{s}, \mathrm{t}}+\mathrm{B}_{\mathrm{t}-1, \mathrm{i}}
$$

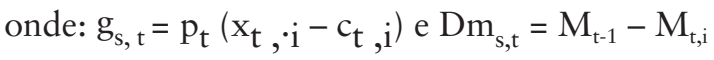

A equação (25) consiste na oferta de crédito ou de empréstimos por parte do indivíduo i. Está claro que para um indivíduo ser ofertante de crédito ele deve vender mais bens do que compra e/ou deve reduzir sua disponibilidade de encaixes monetários ociosos.

Numa economia de troca-pura não há produção de mercadorias, logo não existe investimento em capital fixo. Daqui se segue que todas as obrigações existentes foram emitidas por indivíduos que compram mais mercadorias do que vendem e/ou acumulam saldos monetários inativos. Sendo assim, temos que:

$$
\mathrm{B}_{\mathrm{t}, \mathrm{i}}=\mathrm{gd}, \mathrm{t}+\mathrm{DM}_{\mathrm{d}, \mathrm{t}}+\mathrm{B}_{\mathrm{t}-1, \mathrm{i}}
$$


onde: $\mathrm{g}_{\mathrm{d}, \mathrm{t}}=\mathrm{p}_{\mathrm{t}}\left(\mathrm{c}_{\mathrm{t}, \mathrm{i}}-\mathrm{x}_{\mathrm{t}, \mathrm{i}}\right)$ e $\mathrm{DM}_{\mathrm{d}, \mathrm{t}}=\mathrm{M}_{\mathrm{t}, \mathrm{i}}-\mathrm{M}_{\mathrm{t}-\mathrm{1}}$

Somando (25) e (26) para os $n$ indivíduos, temos:

$$
\begin{aligned}
& B_{d, t}=G_{s, t}+D_{s, t}+B_{t-1} \\
& B_{s, t}=G_{d, t}+D_{d, t}+B_{t-1}
\end{aligned}
$$

onde: $\mathrm{B}_{\mathrm{d}, \mathrm{t}}$ é a demanda agregada por obrigações; $\mathrm{B}_{\mathrm{s}, \mathrm{t}}$ é a oferta agregada de obrigações; $\mathrm{G}_{\mathrm{s}, \mathrm{t}}$ é a oferta agregada de bens; $\mathrm{G}_{\mathrm{d}, \mathrm{t}}$ é a demanda agregada de bens.

Subtraindo (27a) de (27b) temos que:

$$
\left(\mathrm{B}_{\mathrm{d},}-\mathrm{B}_{\mathrm{s}, \mathrm{t}}\right)=\left(\mathrm{G}_{\mathrm{s}, \mathrm{t}}-\mathrm{G}_{\mathrm{d}, \mathrm{t}}\right)+\left(\mathrm{DM}_{\mathrm{s}, \mathrm{t}}-\mathrm{DM}_{\mathrm{d}, \mathrm{t}}\right)
$$

De (28) temos que se dois mercados estão em equilíbrio, o terceiro também estará. Trata-se da famosa Lei de Walras, que nos permite eliminar uma das equações do modelo em consideração, sem afetar a determinação dos preços relativos e da taxa de juros. Senso assim, podemos eliminar tanto a equação de equilíbrio do mercado de obrigações $\left(\mathrm{B}_{\mathrm{d}, \mathrm{t}}=\mathrm{B}_{\mathrm{s}, \mathrm{t}}\right)$ como a referente ao equilíbrio no mercado monetário $\left(\mathrm{DM}_{\mathrm{d}, \mathrm{t}}=\mathrm{DM}_{\mathrm{s}, \mathrm{t}}\right)$.

Até aqui, os resultados são idênticos aos obtidos por Hicks. O ponto novo levantado por Patinkin é que tais resultados não se alteram no caso em que consideramos os fluxos demandados e ofertados de obrigações ao invés dos estoques demandados e ofertas das mesmas.

De fato, subtraindo $B_{t, 1}$ de $B_{d, t}$ e de $B_{s, t}$, temos:

$$
\begin{aligned}
& \mathrm{DB}_{\mathrm{d}, \mathrm{t}}=\mathrm{B}_{\mathrm{d}, \mathrm{t}}-\mathrm{B}_{\mathrm{t}-1} \\
& \mathrm{DB}_{\mathrm{s}, \mathrm{t}}=\mathrm{B}_{\mathrm{s}, \mathrm{t}}-\mathrm{B}_{\mathrm{t}-1}
\end{aligned}
$$

De (29a) e (29b) se os estoques demandados e ofertados de obrigações, para o final do período, forem iguais entre si, segue-se que também haverá equilíbrio entre os fluxos demandados e ofertados das mesmas. Sendo assim, podemos igualmente trabalhar com a condição de equilíbrio do mercado de obrigações escrita em termos de estoques ou em termos de fluxos. Em verdade, se subtrairmos $B_{t, 1}$ de (29a) e (29b), e procedermos à subtração das equações resultantes, temos que:

$$
\mathrm{DB}_{\mathrm{d}, \mathrm{t}}-\mathrm{DB}_{\mathrm{s}, \mathrm{t}}=\left(\mathrm{G}_{\mathrm{s}, \mathrm{t}}-\mathrm{G}_{\mathrm{d}, \mathrm{t}}\right)+\left(\mathrm{DM}_{\mathrm{s}, \mathrm{t}}-\mathrm{DM}_{\mathrm{d}, \mathrm{t}}\right)
$$

De (30) chegamos a um resultado análogo ao que havíamos chegado em (2): pode-se eliminar tanto a equação de equilíbrio do mercado de obrigações como a equação de equilíbrio do mercado de moeda que os preços relativos e a taxa de juros não irão se alterar. Daqui se conclui que a dicotomia estoques-fluxos é irrelevante para a questão da determinação da taxa de juros.

Em resumo, Patinkin afirma que a dicotomia estoques-fluxos é irrelevante para o problema da determinação da taxa de juros, uma vez que se pode provar que equilíbrio de estoques implica equilíbrio de fluxos e vice-versa. Sendo assim, a condição de equilíbrio dos mercados de moeda e obrigações podem ser escritas igualmente em termos de estoques ou de fluxos. A aplicação da Lei de Walras 
permite que qualquer uma dessas condições de equilíbrio seja eliminada do modelo sem que se altere os preços relativos e a taxa de juros de equilíbrio.

\section{UMA AVALIAÇÃO DAS TENTATIVAS DE DEMONSTRAÇÃO DA EQUIVALÊNCIA ENTRE A T.F.E. E A T.P.L.}

É chegado o momento de se passar a avaliação das tentativas de demonstração da equivalência entre a T.F.E. e a T.P.L. Em primeiro lugar, devemos observar que a maior parte dessas tentativas partiram de visões equivocadas a respeito dos pontos em debate na polémica entre Keynes e os defensores da T.F.E. Em particular, as demonstrações de Hicks e Patinkin baseiam-se no pressuposto de que o que está em jogo no dito debate é o mercado no qual a taxa de juros é determinada ou a dicotomia estoques-fluxos. $\mathrm{Na}$ verdade, o que está em jogo é o mecanismo pelo qual as decisões de poupança e investimento influenciam a determinação da taxa de juros.

No que se refere as demonstrações de Lerner e Tsiang, pode-se afirmar que as mesmas não foram bem-sucedidas por não levar em conta alguns dos aspectos essenciais da T.F.E. e/ou da T.P.L. Lerner considerou que a T.F.E. afirmava que a taxa de juros seria determinada, não pelos planos de poupança e investimento, mas pelas magnitudes realizadas dessas variáveis. Por outro lado, Tsiang não tratou de forma adequada o motivo finanças de demanda de moeda, desconsiderando o ponto levantado por Keynes de que a demanda de moeda devida ao motivo finanças seria atendida por um fundo rotativo de ativos líquidos.

De fato, a demonstração da equivalência dada por Lerner está alicerçada na identidade entre poupança e investimento. Como foi observado anteriormente, essa identidade não se origina da definição dos termos poupança e investimento, tal como foi sugerido por Robertson (cf. Presley, 1978, p. 166), mas sim do fato de que tanto Keynes como Lerner considerarem a poupança e o investimento ex-post, ao invés da poupança e do investimento planejados ou ex-ante.

Os economistas da tradição dos modelos de fundos de empréstimo sempre consideraram que os conceitos de poupança e investimento relevantes para a análise da determinação da taxa de juros seriam a poupança e o investimento planejados pelas famílias e pelas firmas. A determinação das variáveis econômicas é feita com base nas escolhas que estão disponíveis aos indivíduos; não a partir de identidades contábeis. Nas palavras de Robertson:

“(...) Analysis deal in terms of the way in which people are willing to act at a particular time it is $(\ldots)$ necessary to interpret the terms used in a sense which makes it possible to relate then to the choices which are open to people at that time" (Cf. Robertson, 1940, pp. 14-5)

"Mr. Lerner, in a recent geometrical fantasia, appears to me to have overlooked these considerations. Starting with an apparatus designed to register human choices, he proceeds to graft on to it concepts from which choice is excluded" (ibid., p. 15, $\mathrm{n}^{\circ} 1$ ). 
Se os conceitos de poupança e investimento relevantes para a teoria de fundos de empréstimo são os conceitos ex-ante, segue-se que a demonstração de Lerner de equivalência entre as duas teorias não é válida.

De forma análoga, a demonstração de equivalência dada por Tsiang também não é válida, porque o mesmo não representa adequadamente o motivo finanças de demanda por moeda. Segundo Keynes a demanda por moeda devida ao motivo finanças seria atendida por um fundo rotativo - "revolving fund" - de ativos líquidos (moeda e crédito bancário), gerenciado pelos bancos comerciais (CWJMK, vol. XIV, p. 219). A demanda por liquidez, devida aos empresários que desejam investir no futuro próximo, pode ser satisfeita pelos encaixes ociosos que os empresários, que já efetivaram os gastos com seus projetos de investimento, tornam a recolocar na circulação ativa. Dito de outra forma: se existem, num dado ponto do tempo, empresários que desejam ficar mais líquidos para poderem efetivar futuramente seus projetos de investimento; existem outros que estão reduzindo o seu grau de liquidez, por estarem implementando seus projetos de investimento. Sendo assim, a demanda líquida por moeda se manterá inalterada; a não ser que o investimento agregado esteja crescendo ao longo do tempo, impondo uma demanda não estacionária por finance. Nas palavras de Keynes:

“(...) Finance is a revolving fund. In the main the flow of new finance required by current ex ante investment is provided by the finance released by current ex post investment. When the flow of investment is at steadystate, so that the flow of ex ante investment is equal to the flow of ex post investment, the whole of it can be provided in this way without any change in the liquidity position" (CWJMK, vol. XIV, p. 220).

Com base nessas considerações a função demanda por moeda deveria ser especificada da seguinte maneira:

$$
\mathrm{M}_{\mathrm{d}, \mathrm{t}}=\mathrm{L}\left(\mathrm{C}_{\mathrm{p}, \mathrm{t}}+\mathrm{I}_{\mathrm{p}, \mathrm{t}}-\mathrm{C}_{\mathrm{t}, 1}-\mathrm{I}_{\mathrm{t}-1} ; \mathrm{r}_{\mathrm{t}}\right)
$$

Em palavras, a demanda por moeda é influenciada pela diferença entre os gastos planejados para o período t e os gastos implementados no período t-1; uma vez que, se os gastos planejados em $\mathrm{t}$ e os efetivados em $\mathrm{t}-1$ forem iguais, o efeito dos mesmos sobre a demanda líquida de moeda será nulo.

Com base em (31), a condição de equilíbrio no mercado monetário pode ser apresentada pela seguinte equação:

$$
\mathrm{M}_{\mathrm{t}}=\mathrm{L}\left(\mathrm{C}_{\mathrm{p}, \mathrm{t}}+\mathrm{I}_{\mathrm{p}, \mathrm{t}}-\mathrm{C}_{\mathrm{t}-1}-\mathrm{I}_{\mathrm{t}-1}, \mathrm{r}_{\mathrm{t}}\right)
$$

Consideremos, agora, que os indivíduos, com o objetivo de financiar seus gastos planejados de consumo para o período $t$, retêm a totalidade da renda que obtiveram ao final do período t- $1^{4}$. Nesse caso, $\mathrm{C}_{\mathrm{p}, \mathrm{t}}$ na equação (32) deve ser substituído por $\mathrm{Y}_{\mathrm{t}-1}$. Temos, portanto, que:

\footnotetext{
${ }^{4}$ Essa hipótese se justifica pelo fato de que os indivíduos não decidem em ta forma pela qual irão manter
} 


$$
\mathrm{M}_{\mathrm{t}}=\mathrm{L}\left(\mathrm{Y}_{\mathrm{t}-1}+\mathrm{I}_{\mathrm{p}, \mathrm{t}}-\mathrm{Y}_{\mathrm{t}-2}-\mathrm{I}_{\mathrm{t}-1} ; \mathrm{r}_{\mathrm{t}}\right)
$$

Linearizando a equação (33) por Taylor em torno de $\mathrm{r}_{\mathrm{t}-1}$, desconsiderando os termos com ordem superior a um e supondo que a função de demanda por moeda é linear na variação dos gastos planejados, temos que:

$$
\mathrm{M}_{\mathrm{t}}=\mathrm{K}\left(\mathrm{r}_{\mathrm{t}-1}\right)\left[\mathrm{Y}_{\mathrm{t}-1}+\mathrm{I}_{\mathrm{p}, \mathrm{t}}-\mathrm{Y}_{\mathrm{t}-2}-\mathrm{I}_{\mathrm{t}-1}\right]+\mathrm{L}_{\mathrm{r}}\left(\mathrm{r}_{\mathrm{t}}-\mathrm{r}_{\mathrm{t}-1}\right)
$$

Defasando-se a equação (34) em um período e subtraindo a resultante de (34), temos que:

$$
\mathrm{DM}_{\mathrm{t}}=\mathrm{K}\left(\mathrm{r}_{\mathrm{t}-1}\right)\left[\left(\mathrm{DY}_{\mathrm{t}-1}-\mathrm{DY}_{\mathrm{t}-2}\right)+\left(\mathrm{DI}_{\mathrm{t}}-\mathrm{DI}_{\mathrm{t}-1}\right)\right]+\mathrm{L}_{\mathrm{r}}\left(\mathrm{r}_{\mathrm{t}}-\mathrm{r}_{\mathrm{t}-1}\right)
$$

Em (35) se observa que a taxa de juros no período $t$ depende, entre outros fatores, da diferença entre o investimento planejado para o período t e o investimento realizado no período anterior. Daqui se segue que a incorporação do motivo finanças de demanda de moeda não é condição suficiente para demonstrar a equivalência entre a T.F.E. e a T.P.L.; pois se para a primeira os niveis planejados de poupança e investimento tem influência direta sobre a taxa de juros - como se observa claramente na equação (23) - na segunda é a diferença entre o investimento planejado para um período e o investimento realizado no período anterior é que tem influência direta sobre a taxa de juros.

\section{CONCLUSÃO}

Ao longo deste artigo observamos que as tentativas de demonstração da equivalência entre a T.F.E. e a T.P.L. feitas por Hicks, Lerner, Tsiang e Patinkin não foram bem sucedidas em seu intento. Isso se deve a dois motivos básicos. Em primeiro lugar, as tentativas de Hicks e Patinkin partiram de concepções equivocadas a respeito das questões em debate entre os defensores da T.F.E. e os defensores da T.P.L. Para Keynes e Robertson o foco de disputa entre a T.F.E. e a T.P.L. era o mecanismo pelo qual as decisões de poupança e investimento influenciam na determinação da taxa de juros. Tal ponto não foi compreendido por Hicks e Patinkin. De fato, Hicks interpreta a oposição entre a T.F.E. e a T.P.L. como um debate a respeito do mercado no qual a taxa de juros é determinada; ao passo que Patinkin considera que o essencial nesse debate seria a dicotomia estoques-fluxos.

Em segundo lugar, as tentativas de demonstração de Lerner e Tsiang partem de representações inadequadas da T.F.E. e da T.P.L. Com efeito, Lerner desconsidera o fato de que os conceitos de poupança e investimento relevantes para a T.F.E. são os conceitos de poupança e investimento ex-ante, e não ex-post, de forma que a identidade contábil entre poupança e investimento não pode ser utilizada como crítica

as poupanças obtidas nesse período; ou seja, irão manter as mesmas sob a forma de encaixes monetários até o próximo período, quando serão incorporadas ao estoque de riqueza, tornando-se objeto de decisão de portfólio. 
a T.F.E. Por outro lado, Tsiang ignora o fato de que a demanda por moeda devido ao motivo finanças é atendida por um fundo rotativo de ativos líquidos, de forma que a demanda de moeda deve ser especificada como uma função da diferença entre o investimento planejado para o período corrente e o investimento realizado no período anterior. Nesse contexto, prova-se que a T.P.L. não é equivalente a T.F.E.

\section{BIBLIOGRAFIA}

CARVAL HO, F. C. (1 99 4). “Sorting the Issues Out: the two debates (19 36 /3 7; 1983/86) on Keynes' Finance Motive Revisited”. Anais do XXII Encontro Nacional de Economia. Florianopólis.

HARRIS, L. (1981). Teoria Monetaria. Fondo de Cultura, México.

HICKS, J. R. (1 987). Valor e Capital. Nova Cultural, São Paulo [tradução da edição original de 1939].

KEYNES, J. M. (1973). The General Theory of Employment, Interest and Money. Macmillan, Londres [edição original: 1936]

KEYNES, J. M. (1937a). “The Theory of the Rate of Interest”, in The Collected Writings of John Maynard Keynes. Macmillan, Londres, vol. XIV.

KEYNES, J. M. (1937b). "Alternative Theories of the Rate of Interest”, in The Collected Writings of John Maynard Keynes. Macmillan, Londres, vol. XIV

KEYNES, J. M. (1937c) “The 'Ex Ante' Theory of the Rate of Interest”, in The Collected Writings of John Maynard Keynes. Macmillan, Londres, vol. XIV.

KEYNES, J. M. (1938). "Mr Keynes and Finance”, in The Collected Writings of John Maynard Keynes. Macmillan, Londres, vo l. XIV.

KEYNES, J. M. (1973). The Collected Writings of John Maynard Keynes. Macmillan, Londres, vol. XIII XIV [no texto CWJMK].

LERNER, A. (1947a). "Savings Equals Investment”, in Harris, S (org.). The New Economics. Dennis Dobson, Londres.

LERNER, A. (1947b). "Alternative Formulations of the Theory of Interest", in Harris, S (org.). The New Economics. Dennis Dobson, Londres.

OREIRO, J.L (1996). “Flexibilidade Salarial, Taxa de Juros e Preferência pela Liquidez: alguns ensaios em economia Keynesiana”. Dissertação de Mestrado, PUC-RJ.

OREIRO, J.L (1999a). "A Importância da Separação entre as Decisões de Consumo-Poupança e de Composição de Portfólio para a Determinação da Taxa de Juros: Uma Reavaliação da Equivalência entre a Teoria dos Fundos de Empréstimos e a Teoria da Preferência pela Liquidez”. Revista Brasileira de Economia, vol. 53, $\mathrm{n}^{\circ} 3$.

OREIRO, J.L (1999b). “O Debate sobre os Determinantes da Taxa de Juros”, in Lima, G.T et a/li (orgs.) Macroeconomia Moderna: Keynes e a Economia Contemporânea. Campus, Rio de Janeiro.

PATINKIN, D. (1958). “Liquidity Preference and Loanable Funds: Stock and Flow Ana lysis”. Economica, 25.

PRESLEY, J.R (1978). Robertsonian Economics. Macmillan, Londres.

ROBERTSON, D. (1940). Essays in Monetary Theory. P. S.King, Londres.

TSIANG, S.C (1956) "Liquidity Preference and Loanable Funds Theories, Multiplier and Velocity Analyses: a synthesis". American Economic Review, vol. XLVI, nº 4.

TSIANG, S.C (1980). “Keynes's Finance Demand for Liquidity, Robertson's Loanable Funds Theory and Friedman's Monetarism”. The Quarterly Journal of Economics, Maio. 